\section{Some considerations on de Waal and Preston review}

\author{
Giacomo Rizzolatti and Fausto Caruana
}

In their interesting Review, de Waal and Preston propose that the emotional states of others are "understood through personal, embodied representations that allow empathy" (Nat. Rev. Neurosci. 18, 498-509 (2017)) $)^{1}$. One of the key pieces of neuroscientific evidence for such a proposal has been the discovery of mirror neurons in the monkey parietal and premotor areas. Despite recognizing that the mirror mechanism plays an important role in various aspects of affective empathy ${ }^{1}$, the authors suggest that this mechanism is only a part of a "bigger picture" of empathy, with the mirror mechanism being restricted to the motor system. Other aspects of empathy are linked to the mirror mechanism indirectly, and are better explained by the perceptionaction model (PAM).

The ability to perform intracranial recordings in humans and then to stimulate the recorded sites in the same patients demonstrated the presence of the mirror mechanism in centres responsible for emotions, thus extending its role outside of the parieto-frontal motor centres. Criteria for claiming that an area is endowed with the mirror mechanism for empathy are the following: electrical stimulation must produce a specific emotion; presentation of social stimuli that show this same emotion should produce a modulation of neural activity in these sites; and, in addition, lesions to the same area should produce impairment in feeling the emotion and in processing the same emotion in others. If these criteria are satisfied, we have three brain areas endowed with the mirror mechanism that automatically transforms sensory representation of a given emotional state, both in terms of feeling that emotion and in terms of the action tendencies associated with this state.
The first is the anterior insula (AI). Stimulation of the ventral AI produces the feeling of disgust in humans ${ }^{2}$ and monkeys ${ }^{3}$; administration of disgusting stimuli (natural stimuli), as well as observation of facial expression of disgust (social s timuli), activate $\mathrm{AI}^{2,4}$; permanent or temporary lesions to AI impair the capacity of feeling disgust and of recognizing it in others, while the capacity to recognize other emotions remains intact ${ }^{5,6}$.

The second is the amygdala (AM), which produces fear when stimulated in humans ${ }^{7,8}$; the presentation of fearful facial expressions triggers faster and stronger responses relative to other facial expressions ${ }^{9,10}$; selective bilateral destruction of AM (Urbach-Wiethe disease) produces an impaired, abnormal experience of fear ${ }^{11,12}$. These patients do not feel fear but are able to describe cognitively what fear is ${ }^{13}$.

The third area is the perigenual sector of anterior cingulate cortex (pACC). Stimulation of pACC elicits smiling and laughter often with mirth $^{14}$, and the presentation of movies showing laughing, but not crying or neutral expressions, modulates the gamma band activity in the same site ${ }^{15}$.

These data strongly support the contention that empathy is based on personal, embodied representations of emotions that are mediated by the mirror mechanism. We argue that this mechanism is not complementary to the PAM, nor a subpart of it; rather, in the light of new emerging studies in the field of human electrophysiology, the mirror mechanism appears to be the principal component responsible for the "overlapping neural signature for experiencing and observing affective states".
There is a reply to this Correspondence from Preston, S. D. and de Waal, F. B. M. Nat. Rev. Neurosci. http://dx.doi.org/10.1038/ nrn.2017.140 (2017).

Giacomo Rizzolatti is at the Dipartimento di Medicina e Chirurgia, Università di Parma, Italy; and at Istituto di Neuroscienze, Consiglio Nazionale delle Ricerche, URT Parma, I-43100 Parma, Italy.

Fausto Caruana is at the Istituto di Neuroscienze, Consiglio Nazionale delle Ricerche, URT Parma, I-43100 Parma, Italy.

Correspondence to G.R. giacomo.rizzolatti@unipr.it doi: $10.1038 / \mathrm{nrn} .2017 .139$ Published online 3 Nov 2017

1. de Waal, F. B. M. \& Preston, S. D. Mammalian empathy: behavioural manifestations and neural basis. Nat. Rev. Neurosci. 18, 498-509 (2017).

2. Krolak-Salmon, P. et al. An attention modulated response to disgust in human ventral anterior insula. Ann. Neurol. 53, 446-453 (2003).

3. Jezzini, A. et al. Functional organization of the insula and inner perisylvian regions. Proc. Natl Acad. Sci. USA 109, 10077-10082 (2012).

4. Gallese, V. et al. A unifying view of the basis of social cognition. Trends Cogn. Sci. 8, 396-403 (2004).

5. Calder, A. J. et al. Impaired recognition and experience of disgust following brain injury. Nat. Neurosci. 3, 1077-1078 (2000)

6. Papagno, C. et al. Specific disgust processing in the left insula: new evidence from direct electrical stimulation. Neuropsychologia 84, 29-35 (2016).

7. Meletti, S. et al. Emotions induced by intracerebral electrical stimulation of the temporal lobe. Epilepsia 47, 47-51 (2006).

8. Lanteaume, L. et al. Emotion induction after direct intracerebral stimulations of human amygdala. Cereb. Cortex 17, 1307-1313 (2007).

9. Méndez-Bértolo, C. et al. A fast pathway for fear in human amygdala. Nat. Neurosci. 19, 1041-1049 (2016).

10. Meletti, S. et al. Fear and happiness in the eyes: an intra-cerebral event-related potential study from the human amygdala. Neuropsychologia 50, 44-54 (2012).

11. Meletti, S. et al. Temporal lobe epilepsy and emotion recognition without amygdala: a case study of Urbach-Wiethe disease and review of the literature. Epileptic Disord. 16, 518-527 (2014).

12. Adolphs, R. et al. Impaired recognition of emotion in facial expressions following bilateral damage to the human amygdala. Nature 372, 669-672 (1994).

13. Damasio, A. R. The Feeling of What Happens: Body and Emotion in the Making of Consciousness (Harcourt Brace, 1999).

14. Caruana, F. et al. Mirth and laughter elicited by electrical stimulation of the human anterior cingulate cortex. Cortex 71, 323-331 (2015).

15. Caruana, F. et al. A mirror mechanism for smiling in the anterior cingulate cortex. Emotion 17, 187-190 (2016).

Competing interests statement

The authors declare no competing interests. 